\title{
Conjugated Molecules for the Smart Filtering of Intense Radiations
}

\section{Danilo Dini}

Institut für Organische Chemie, University of Tübingen, Auf der Morgenstelle 18, 72076 Tübingen, Germany. Phone:+49-7071-29-78744; Fax:+49-7071-29-5268; E-mail: danilo.dini@uni-tuebingen.de

Received: 23 January 2003 / Accepted: 4 March 2002 / Published: 15 April 2003

\begin{abstract}
The practical realization of smart optical filters, i.e. devices which change their optical transmission in a suitable way to keep a working state for a general light sensitive element, can involve the use of conjugated molecules whose light absorption properties are light- intensity dependent (nonlinear optical effect). The verification of optical limiting displayed by some particular conjugated molecules, e.g. phthalocyanines, is quite noteworthy and can be successfully exploited for the realization of such smart optical devices. In the present contribution the analysis of the relevant molecular feature of a phthalocyanine are analyzed with the aim of determining useful correlations between optical limiting performance and phthalocyanine chemical structure. In particular, the electronic nature of the substituent is considered as a key factor for the explanation of some observed optical limiting trends.
\end{abstract}

Keywords: Conjugated molecule, smart optical filter, intensity dependent optical properties.

\section{Introduction}

The smart filtering of a luminous radiation is a complex process whose realization implies the controlled modulation of the optical properties of those device elements designed to interact with the luminous radiation. Such a process of smart optical filtering represents a non trivial technological achievement due to the increasing use of the light as fast vehicle for the transmission of signals and energy, which requires continuously the development of adequate systems for the processing of light [1]. Depending on the nature of the luminous radiation, i.e. natural, artificial, accidental or hostile, the design of a suitable smart optical filter represents an interdisciplinary task which needs contributions 
equally from optics to electronics, from materials science to physical chemistry and synthetic chemistry among others. In the present context the term "smart" refers to those systems capable to modify opportunely their optical properties when interacting with the luminous radiation, in order to control the radiation in a convenient way [2]. Smart optical devices employ generally materials whose optical properties are varied by means of an electric current as in electrochromic devices [3], or via the absorption of radiations with a characteristic spectrum like in the case of photochromic devices [4]. A further way of modulating the optical properties of materials is offered by nonlinear optical (NLO) effects which consist of the change of the optical properties of materials induced by the high intensity of the interacting radiation [5]. High intensity radiations are practically generated only by lasers [6] and due the continuously increasing use of these artificial light sources in the most disparate applications ranging from image formation in holography to data transfer in optical computer or heat generation in high-temperature production processes [7-10], it appears strongly motivated the study of materials with NLO properties for the possible control and manipulation of laser radiations. In particular, the occurrence of NLO effects can be properly exploited for the creation of special filters possessing optical limiting (OL) features, i.e. systems whose overall light-absorbance increases with the increase of the incident light intensity [11]. Such a NLO effect finds an important application in the protection of all those light sensors which must operate below a safety threshold of the incident intensity to prevent unrecoverable laser damage [12]. A prominent class of materials displaying NLO absorption and concomitant generation of the OL effect is constituted by conjugated organic molecules [13] which are characterized by the presence of an extended network of loosely bound $\pi$-electrons with oxidation energies ranging within the approximate interval 6.5-8.5 eV [14]. The most relevant feature of the $\pi$-electrons is their high polarizability $[15,16]$. This allows a quasi-instantaneous response of the molecule to the perturbations induced by rapidly variable external electric fields and the interaction between light and conjugated molecule generally brings about low losses and scarce dispersion of the energy transported by the optical wave [5]. The property of interest of the material used for the description of the NLO effects produced by the irradiation of the material with a laser light source is commonly the vector polarization $\boldsymbol{P}$ defined as the number of dipole moments per unit volume of material (in $\mathrm{C} \mathrm{m}^{-2}$, in MKS). Polarization $\boldsymbol{P}$ is related with the applied electric field $\boldsymbol{E}$ (in $\mathrm{V} \mathrm{m}^{-1}$, in MKS) of the radiation through the relationship:

$$
\boldsymbol{P}=\varepsilon_{0} \chi \boldsymbol{E}
$$

where $\chi$ is the generalized susceptibility, and $\varepsilon_{0}$ the dielectric constant of the vacuum $\left(8.85 \times 10^{-12} \mathrm{~F}\right.$ $\mathrm{m}^{-1}$ ). In the case of irradiation with strong applied fields $\boldsymbol{E}$, the susceptibility $\chi$ becomes itself a function of $\boldsymbol{E}$, i.e. $\chi(\boldsymbol{E})$, thus giving rise to a nonlinear dependence of $\boldsymbol{P}$ on $\boldsymbol{E}$. The OL effect relies on the variation of the generalized nonlinear absorption coefficient $\alpha_{\mathrm{NL}}\left(\mathrm{in} \mathrm{m}^{-1}\right.$ ) with the incident light 
intensity I (in $\mathrm{W} \mathrm{m}^{-2}$ ) according to the modified Lambert-Beer law [13]:

$$
(d I / d z)=-\alpha_{N L}(I) I
$$

where $\mathrm{z}$ is the direction of light propagation through the material. The coefficient $\alpha_{\mathrm{NL}}$ is given by the approximate relationship:

$$
\alpha_{\mathrm{NL}}=\alpha+\beta \mathrm{I}
$$

with $\alpha$ (in $\mathrm{m}^{-1}$ ) and $\beta$ (in $\mathrm{m} \mathrm{s} \mathrm{J}^{-1}$ ) corresponding to the linear absorption coefficient and the secondorder nonlinear absorption coefficient, respectively.

The correlation between the generalized optical susceptibility in Equation 1, with the second-order nonlinear absorption coefficient is given by the relationship [17]:

$$
\operatorname{Im}\left[\chi^{(3)}\right]=\left(2 \mathrm{c}^{2} \mathrm{n}_{0}^{2} \beta / \pi \omega *\right) \times 10^{-22} \mathrm{C} \mathrm{m} \mathrm{V}^{-3}
$$

where $\chi^{(3)}$ is the third-order susceptibility, $\mathrm{c}$ is the speed of light $\left(3 \times 10^{8} \mathrm{~m} \mathrm{~s}^{-1}\right), \mathrm{n}_{0}$ is the linear refractive index of the system, and $\omega *$ is the incident light frequency.

In the framework of the OL effect generated by molecular systems like organic conjugated species, relatively few attempts have been made to find precise correlations between molecular structure and corresponding OL effect [18]. In the following the analysis and the discussion of the results concerning the OL effect produced by some conjugated macrocycle-metal complexes are reported with the aim of correlating the features of the molecular structure with the intensity-dependent effect of reverse saturable absorption [19] in the perspective of a possible involvement in the realization of practical smart optical limiters.

\section{Experimental}

\section{Optical Limiting Measurements}

The OL effect for the various conjugated macrocycle-metal complexes has been studied with the Zscan technique in the open aperture configuration [20]. Such a configuration allows the determination of the NLO effects associated solely with the changes of the nonlinear absorption coefficient and not with the changes of the nonlinear refractive index. The samples were solutions of conjugated macrocycle-metal complexes in 1-chloronaphthalene with concentrations ranging in the interval 0.5 $5 * 10^{-4} \mathrm{M}$. The optical cell was a quartz cuvette with optical path thickness equal to $0.1 \mathrm{~cm}$. The sample irradiation was realized with a pulsed Nd:YAG laser (from Continuum Minilite, Edinburgh, Scotland) 
emitting at the second harmonic $(\lambda=532 \mathrm{~nm})$. Samples linear transmittance at the wavelength of laser irradiation ranged in the interval 85-90\%. The maximum energy per laser pulse was $100 \mathrm{~mJ}$. The pulse duration ranged in the interval 3-7 ns. The frequency of sample irradiation was 10 pulses per second. The Nd:YAG laser beam was focused with a neutral lens having the focal distance equal to $10 \mathrm{~cm}$. During the Z-scan determinations the sample spanned a total length of $3.5 \mathrm{~cm}$ centered at the beam focus. The displacement of the sample along the Z-scan direction was controlled by means of a piezoelectric driver. The laser beam radius was approximately $0.5 \mathrm{~cm}$ before lens focusing. The recording of sample transmittance was taken every $0.05 \mathrm{~cm}$ along the spanned length. The reported values of transmittance were averaged on a basis of twenty determinations per analyzed Z-position.

\section{Synthesis of conjugated macrocycle-metal complexes}

The metal-macrocycle complexes which have been studied for the generation of the OL effect are a series of new phthalocyaninatotitanium(IV) complexes with general formula $\mathrm{R}_{\mathrm{y}} \mathrm{PcTiX}_{\mathrm{n}}$ with $\mathrm{R}$ and $\mathrm{X}$ being respectively the peripheral substituent of the phthalocyanine $(\mathrm{Pc})$ ring and axial substituent at the central metal atom Ti. The choice of $\mathrm{R}_{\mathrm{y}} \mathrm{PcTiX}_{\mathrm{n}}$ species is motivated by the promising OL properties shown by some phthalocyaninatotitanium(IV) complexes characterized by different kinds of axial ligands $\mathrm{X}[21,22]$. The $\mathrm{R}_{\mathrm{y}} \mathrm{PcTiX}_{\mathrm{n}}$ species whose OL properties are here investigated are listed in Table 1 and the references concerning the details of their preparation are given in the last column of the table.

Table 1. List of phthalocyaninatotitanium(IV) oxide studied for OL generation in this work.

\begin{tabular}{|c|c|c|c|c|c|}
\hline Species & $\mathrm{R}$ & $\mathrm{y}$ & $X$ & $\mathrm{n}$ & Ref \\
\hline $\begin{array}{l}\text { Hexadecafluorophthalocyaninatotitanium } \\
\text { oxide }\left[\mathrm{F}_{16} \mathrm{PcTiO}\right](1)\end{array}$ & $\mathrm{F}$ & 16 & $\mathrm{O}$ & 1 & 23 \\
\hline $\begin{array}{l}\text { 2(3)-Tetratertbutylphthalocyaninato- } \\
\text { titanium oxide }\left[\mathrm{t}-\mathrm{Bu}_{4} \mathrm{PcTiO}\right]\end{array}$ & $-\mathrm{C}\left(\mathrm{CH}_{3}\right)_{3}$ & 4 & $\mathrm{O}$ & 1 & 21 \\
\hline $\begin{array}{l}\text { Hexadecachlorophthalocyaninatotitanium } \\
\text { oxide }\left[\mathrm{Cl}_{16} \mathrm{PcTiO}\right] \text { (3) }\end{array}$ & $\mathrm{Cl}$ & 16 & $\mathrm{O}$ & 1 & 24 \\
\hline $\begin{array}{l}\text { Hexadecachlorophthalocyaninatotitanium } \\
\text { dichloride }\left[\mathrm{Cl}_{16} \mathrm{PcTiCl}_{2}\right] \text { (4) }\end{array}$ & $\mathrm{Cl}$ & 16 & $\mathrm{Cl}$ & 2 & 25 \\
\hline $\begin{array}{l}\text { (4-tertButylcatecholato)phthalocyani- } \\
\text { natotitanium oxide }\left[\mathrm{PcTi}\left(\mathrm{O}_{2} \mathrm{C}_{6} \mathrm{H}_{3}\right) \mathrm{t}-\mathrm{Bu}\right] \text { (5) }\end{array}$ & $\mathrm{H}$ & 16 & & 1 & 22 \\
\hline $\begin{array}{l}\text { Tetrapyrazinotetraazaporphyrinato titanium } \\
\text { oxide [Pyr } 4 \text { TAPTiO] (6) }\end{array}$ & $\mathrm{N}$ & 8 & $\mathrm{O}$ & 1 & 26 \\
\hline
\end{tabular}




\section{Results}

The Z-scan profiles for the species (1)-(6) are shown in Figure 1.

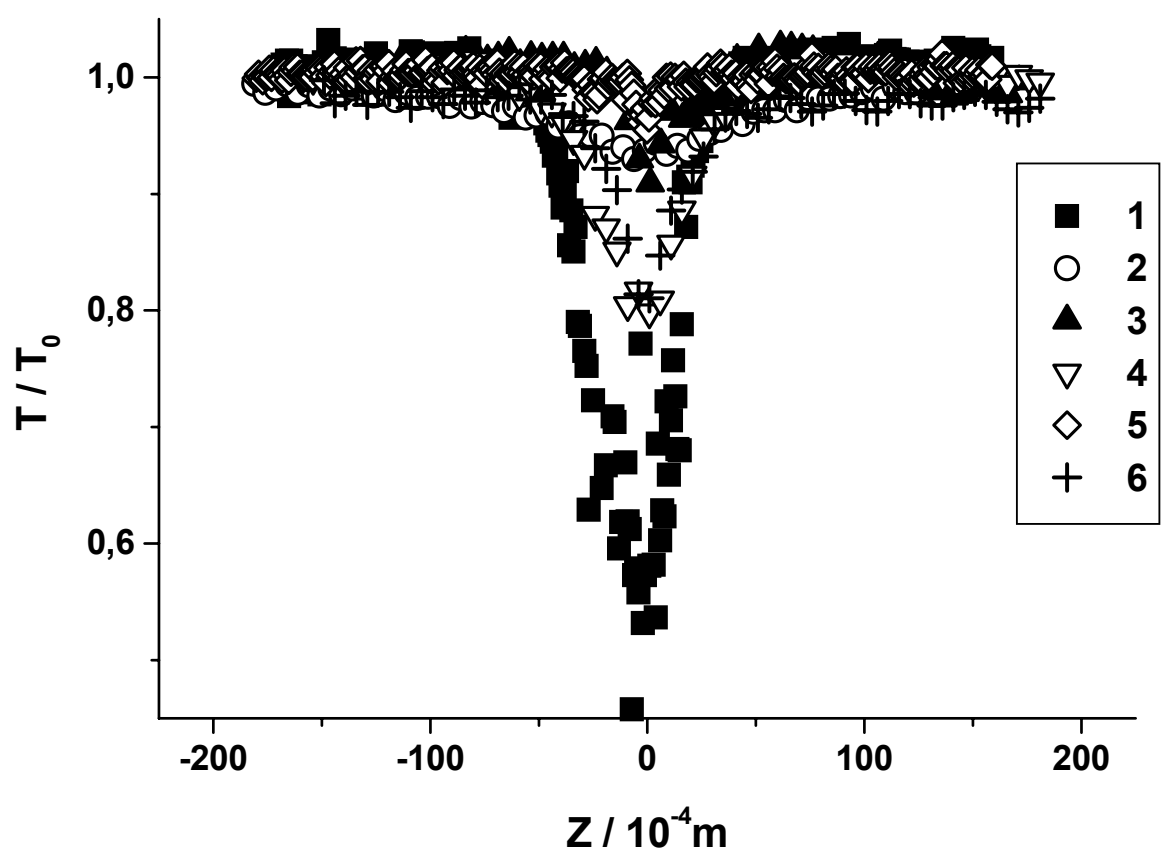

Figure 1. Z-scan profiles for the species (1)-(6). The species were irradiated at the same level. The linear transmittance $\mathrm{T}_{0}$ of the examined solutions was 0.9 . The concentrations were in the range $4-5^{*}$ $10^{-5} \mathrm{M}$. The 1-chloronaphthalene solvent didn't show any NLO behavior at the used level of irradiation.

In Figure 1 the actual (nonlinear) transmission $\mathrm{T}$ of the (1)-(6) solutions varies with the distance $\mathrm{Z}$ from the focus of a gaussian beam whose intensity I varies with $\mathrm{Z}$ according to [20]:

$$
\mathrm{I}(\mathrm{Z})=E / \tau \mathrm{w}^{2}(\mathrm{Z}) \pi
$$

where $E, \tau$ and $\mathrm{w}(\mathrm{Z})$ are the pulse energy (in J), the pulse duration (in s) and the beam radius (in $\mathrm{m}$ ) as a function of the distance from the focus $\mathrm{Z}$, respectively. The radius $\mathrm{w}$ of a gaussian beam depends on the distance $\mathrm{Z}$ from the focus according to the equation [20]:

$$
\mathrm{w}^{2}(\mathrm{Z})=\mathrm{w}_{0}^{2}\left(1+\mathrm{Z}^{2} / \mathrm{Z}_{0}^{2}\right)
$$

in which $\mathrm{w}_{0}$ is the beam waist at the focus and $\mathrm{Z}_{0}$ the diffraction length of the beam defined as the ratio of the area $\mathrm{w}_{0}{ }^{2} \pi$ of the gaussian beam at the focus to the laser beam wavelength $\lambda$. The OL performance of different species can be evaluated and compared in terms of the minimum value of normalized transmittance $\mathrm{T} / \mathrm{T}_{0}$ which is usually achieved in correspondence of the highest level of irradiation, i.e. at the focus $(Z=0)$, if the linear transmittance $T_{0}$ is the same for all the samples under 
examination. The value of $\mathrm{T} / \mathrm{T}_{0}$ at $\mathrm{Z}=0$ grows in the succession $\mathrm{T} / \mathrm{T}_{0}(\mathbf{1})_{\mathrm{Z}=0}<\mathrm{T} / \mathrm{T}_{0}(\mathbf{4})_{\mathrm{Z}=0} \approx \mathrm{T} / \mathrm{T}_{0}(\mathbf{6})_{\mathrm{Z}=0}$ $<\mathrm{T} / \mathrm{T}_{0}(\mathbf{3})_{\mathrm{Z}=0}<\mathrm{T} / \mathrm{T}_{0}(\mathbf{2})_{\mathrm{Z}=0}<\mathrm{T} / \mathrm{T}_{0}(\mathbf{5})_{\mathrm{Z}=0}$ with $\mathrm{F}_{16} \mathrm{PcTiO}$ (1) showing unambiguously the best $\mathrm{OL}$ performance with the lowest value of $\mathrm{T} / \mathrm{T}_{0}(\mathbf{1})_{\mathrm{Z}=0}(=0.55)$ whereas $\left[\mathrm{PcTi}\left(\mathrm{O}_{2} \mathrm{C}_{6} \mathrm{H}_{3}\right) \mathrm{t}-\mathrm{Bu}\right](\mathbf{5})$ and $[\mathrm{t}-$ $\mathrm{Bu}_{4} \mathrm{PcTiO}$ ] (2) present the smallest diminutions of $\mathrm{T} / \mathrm{T}_{0}$ at the focus. From the compared analysis of the results in Figure 1, it is observed that those phthalocyanines carrying electron withdrawing groups (EWG's) like $\mathrm{F}$ in $\mathbf{1}, \mathrm{Cl}$ in $\mathbf{3}$ and $\mathbf{4}$, and $\mathrm{N}$ as heteroatom in the benzenic moiety of $\mathbf{6}$ give rise to the stronger OL effect.

\section{Discussion}

The origin of the nonlinear transmission in phthalocyanines is related mostly with the occurrence of the absorption of an excited state [27], and less preponderant, by the verification of nonlinear refraction [28]. The mechanism of nonlinear absorption in phthalocyanines is successfully interpreted in terms of the four-level model [29]. This model depicts the nonlinear absorber as a system having four main electronic levels involved in the transitions which give rise to an intensity-dependent absorption (Figure 2).

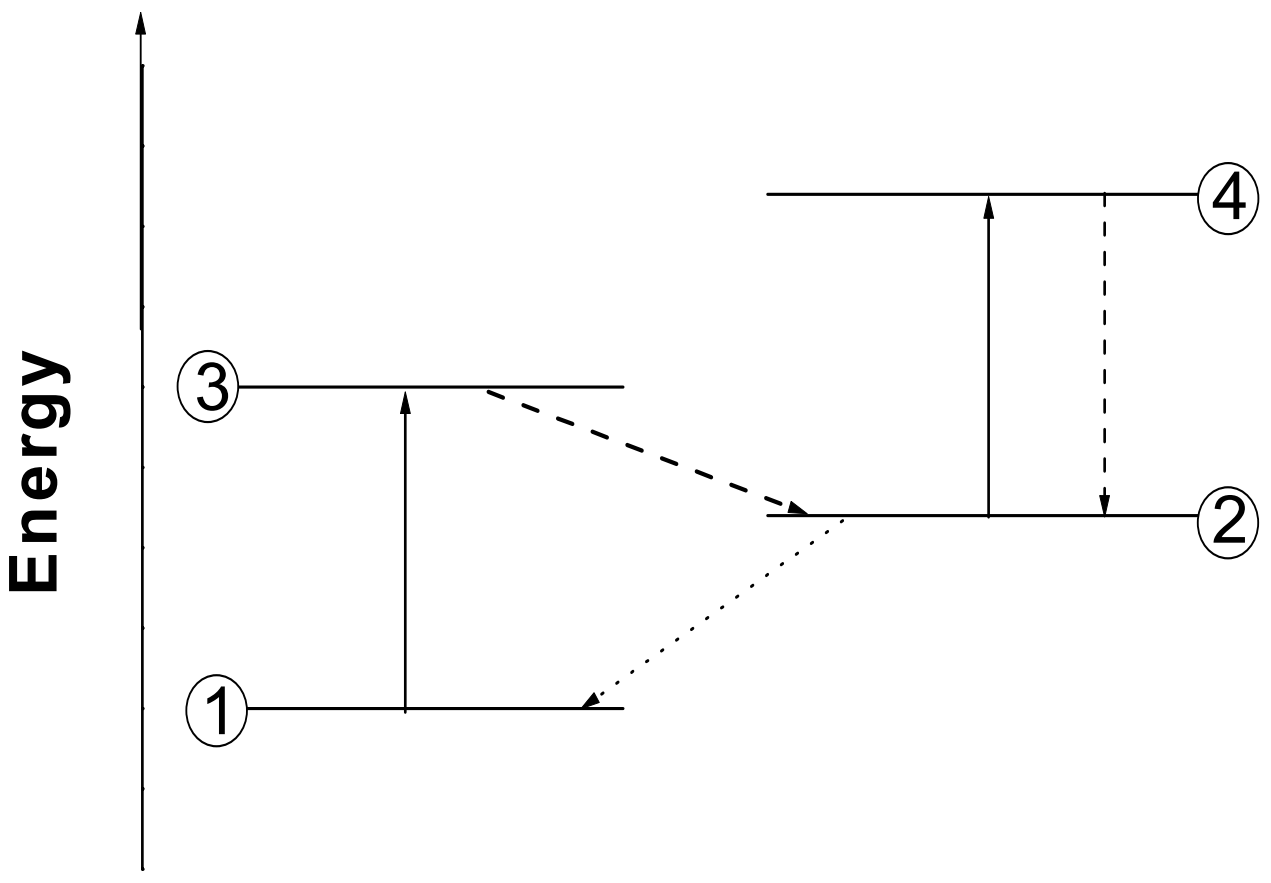

Figure 2. Energy diagram of a four-level system. Full lines indicate absorption from singlet ground $[1 \rightarrow 3]$ and triplet excited states [2 $\rightarrow 4]$; dashed lines represent intersystem crossing [3 $\rightarrow 2]$ and excited state fluorescence $[4 \rightarrow 2]$; dotted line indicates phosphorescence $[2 \rightarrow 1]$. 
In the four-level model the absorptions are due to the consecutive transitions $[1 \rightarrow 3]$ and $[2 \rightarrow 4]$ being $\left(h \omega_{1,3} / 2 \pi\right)=\left(h \omega_{2,4} / 2 \pi\right)$ with $\omega_{i, j}$ indicating the frequency of absorption for the transition $[\mathrm{i} \rightarrow \mathrm{j}]$ and $\mathrm{h}$ the Planck's constant. Therefore, such a mechanism of absorption implies the consecutive absorption of two photons. The intersystem crossing (ISC) transition [3 $\rightarrow 2$ ] (it is supposed that the couples of states 1,3 and 2,4 are respectively singlet and triplet states), and the fluorescence from the upper excited state $[4 \rightarrow 2$ ] are assumed to be fast processes in order to get effective optical limiting. In fact, a fast intersystem crossing combined together with fast fluorescent decay [4 $\rightarrow 2$ ] allows to populate the triplet excited state $i=2$ which represent the initial state of the transition involving the absorption of the second photon. Another necessary condition for the achievement of effective optical limiting is a high value of the absorption cross-section for the transition $[2 \rightarrow 4]\left(\sigma_{24}\right)$ to absorb efficiently the second photon at regimes of high irradiation. It is often convenient to define suitable merit coefficients for evaluating the OL performance of different systems in different conditions. The most frequently used merit coefficients are the ratio $\sigma_{24} / \sigma_{13}[30]$ (equivalent to $\sigma_{\text {exc }} / \sigma_{\mathrm{g}}$ with $\sigma_{\text {exc }}$ and $\sigma_{\mathrm{g}}$ being the excited state and ground state absorption cross-sections, respectively), and the difference $\sigma_{24}-\sigma_{13}[18]$ whose high values are indicative of better OL performances. Both $\sigma_{24}\left(\right.$ or $\left.\sigma_{\text {exc }}\right)$ and $\sigma_{13}$ (or $\sigma_{\mathrm{g}}$ ) are correlated with $\beta$ as defined in equation 3 , through the relationship [17]:

$$
\beta=\sigma_{24} \sigma_{13}\left[\mathrm{~N}_{1}\right] \mathrm{N}_{\mathrm{A}}^{2} \Phi_{\mathrm{ISC}}
$$

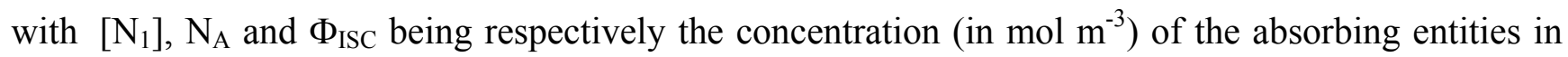
the ground state 1, the Avogadro's number (in $\mathrm{mol}^{-1}$ ) and the ISC efficiency (in mol s J $\mathrm{J}^{-1}$ ) at the working excitation frequency. The use of generalized intensive properties like the absorption crosssection or the molar extinction coefficient does not allow a straightforward correlation between structural parameters of the nonlinear absorber itself and the OL performance. This is because such properties just describe an overall effect with no consideration of the actual phenomenology correlated with the structural features of the active species displaying nonlinear absorption. At this point it would seem more appropriate to accomplish the analysis of the OL performance in terms of the changes of the dipole moments implied in the various transitions associated with the nonlinear absorption processes [31]. This is because a molecular property like the dipole moment can be directly correlated and analyzed in terms of molecular structure features of the active species. Irradiation of phthalocyanines at $532 \mathrm{~nm}$ produces mostly a nonresonant effect consisting of the electronic polarization of the $\pi$-electrons. In fact, phthalocyanines are quasi transparent in the wavelengths window 500-600 nm comprised between the main characteristic Q and B absorption bands, and in correspondence of $532 \mathrm{~nm}$, the closest resonances of phthalocyanines are due to the metal to ligand and/or ligand to metal charge transfer (MLCT/LMCT) processes [32]. The increasing irradiation in this wavelength region induces a growing deformation of the polarizable electronic cloud constituted by the $\pi$-electrons of the phthalocyanine. At a certain point the irradiated molecule reaches a critical state of electronic polarization, which is capable to get in resonance at the wavelength of irradiation. Such a 
resonance of a highly polarized phthalocyanine molecule is believed to involve a MLCT/LMCT process which is red-shifted with respect to the same kind of transition in the ground state [28]. The deformability of the $\pi$-electrons cloud upon irradiation can be expressed as a generalized $\pi$-electrons polarizability d $\left[\rho_{\pi}\left(\mathrm{x}, \mathrm{y}, \mathrm{z}, \boldsymbol{E}_{\text {ext }}\right)\right] / \mathrm{d}\left[\boldsymbol{E}_{\text {ext }}\right]$ where $\rho_{\pi}$ and $\boldsymbol{E}_{\text {ext }}$ are the distribution of the $\pi$-electrons charge over the molecular volume (in $\mathrm{C} \mathrm{m}^{-3}$ ), and the applied perturbating optical field (in $\mathrm{V} \mathrm{m}^{-1}$ ), respectively [33]. Such a rate of deformation depends on the structural features of the molecule like the number of $\pi$-electrons, extension of the conjugated network and the electronic nature of the substituents at the conjugated skeleton [34]. The series of OL curves obtained from the compounds 1-6 (Figure 1), shows a quite well defined trend if the electron withdrawing character of the substituents at the peripheral positions and at the central atom, is considered as key factor in the analysis of these curves. In general, the more increasing the electron-withdrawing character and the number of the EWG's the better is the resulting OL response. Such a correspondence could be explained in terms of strongest optical fields required for the achievement of the critical polarized state in which the phthalocyanine absorbs resonantly the radiation. This effect is a consequence of the increasing stiffness of the electronic cloud due to the presence of EWG's. Another reason of the improvement of the OL effect with EWG's could be also the higher variation of the dipole moment in passing from the absorbing polarized state of the phthalocyanine to the final excited state. This would correspond to higher transition dipole moments in accordance with a correlated increase of the absorption coefficient for that specific transition [35]. Such an analysis leads to conclude that the excited state absorption process involved in the OL phenomenon consists very probably in the transfer of a partial electronic charge from the electron- rich moiety of the metal-macrocycle complex, i.e. the conjugated macrocycle, to the electron-poorer moiety of the complex, i.e. the central metal atom. This gives rise to an intensity-dependent intramolecular reduction which involves the conjugated macrocycle as reducing agent and the central metal atom as oxidizing agent. The effectiveness of this photoinduced ligand to metal charge transfer results higher when the charge separation between the conjugated macrocycle and the central metal is more pronounced in the ground state of the phthalocyanine. Such an intramolecular electronic charge transfer would occur in phthalocyanines at frequencies falling in the approximate range 5-6 $10^{14} \mathrm{~Hz}$ when the intensity of the incident radiation is in the order of $10^{12} \mathrm{~W} \mathrm{~m}^{-2}$.

\section{Conclusions}

The verification of the optical limiting effect in conjugated molecules based systems, is due to the occurrence of intensity-dependent absorption processes which imply the succession of nonresonant and resonant phenomena in the active species. The possible correlation between structural features of a particular class of conjugated molecules for optical limiting, i.e. phthalocyanines, and the optical limiting performance has been attempted by choosing similar chemical structures as terms of comparison. It has been unequivocally found that electron withdrawing substituents at the peripheral 
positions of the macrocycle and at the central metal as axial ligands, produce the molecular systems with the better optical limiting performances. The reasons for such a behavior have been discussed and analyzed in terms of variations of the charge distribution in the several electronic states of the molecule, which are involved in the optical limiting transitions. Such findings could open new directions in the search of more effective molecular systems to be employed as active materials in smart optical filters.

\section{References}

1. Menzel, R. Photonics: Linear and Nonlinear Interactions of Laser Light and Matter; Springer Verlag: Berlin, 2001.

2. Dini, D. Int.J. En. Env. Econ. 2000, 10, 1.

3. Monk, P.M.S.; Mortimer, R. J.; Rosseinsky, D.R. Electrochromism:Fundamentals and Applications, VCH: Weinheim, 1995.

4. Dorion, G. H.; Wiebe, A.F. Photochromism:Optical and Photographic Applications, Focal Press: London, 1970.

5. Boyd, R.W. Nonlinear Optics, Academic Press: Boston, 1991.

6. Maiman, T.H. Nature 1960, 187, 493.

7. Mehta, P.C.; Rampal, V.V. Lasers and Holography, World Scientific: Singapore, 1993.

8. Cerullo, L.J. Application of Lasers in Neurosurgery; Yearbook Medical Publication: Chicago, 1987.

9. Anderberg, B.; Wolbarsht, M.L. Laser Weapons: The Dawn of a New Military Age; Plenum Press: New York, 1992.

10. Schwartz, K.; Shvarts, K.K. The Physics of Optical Recording; Springer Verlag: Berlin, 1994.

11. Geusic, J. E.; Singh, S.; Tipping, D.W.; Rich, T. C. Phys. Rev. Lett. 1967, 19, 1126.

12. Zuclich, J.A.; Lund, D.J.; Edsall, P. R.; Hollins, R. C.; Smith, P. A.; Stuck, B. E.; McLin, L. N. Mol. Cryst. Liq. Cryst. Sci. Techn. Sect. B: Nonlin. Opt. 1999, 21, 19.

13. Hanack, M.; Dini, D.; Barthel, M.; Vagin, S. Chem. Rec. 2002, 2, 129.

14. Bredas, J. L.; Silbey, R.; Boudreaux, D.S.; Chance, R.R. J. Am. Chem. Soc. 1983, 105, 6555.

15. Villesuzanne, A.; Hoarau, J.; Ducasse, L.; Olmedo, L.; Hourquebie, P. J. Chem. Phys. 1992, 96, 495.

16. Cheng, L. T.; Tam, W.; Marder, S.R.; Stiegman, A.E.; Rikken, G.; Spangler, C.W. J. Phys. Chem. 1991, 95, 10643.

17. Prior, Y.; Vogt, H. Phys. Rev. B 1979, 19, 5388.

18. Nalwa, H.S.; Shirk, J.S. In Phthalocyanines: Properties and Applications; Leznoff, C.C.; Lever, A.B.P., Eds.; VCH: New York, 1996, vol.4, p.79.

19. Dini, D.; Barthel, M.; Hanack, M. Eur. J. Org. Chem. 2001, 3759.

20. Sheik-Bahae, M.; Said, A.; Wei, T.; Hagan, D.J.; Van Stryland, E.W. IEEE J. Quantum Electron., 1990, 26, 760.

21. Barthel, M.; Dini, D.; Vagin, S.; Hanack, M. Eur. J. Org. Chem. 2002, 3756. 
22. Barthel, M.; Hanack, M. J. Porphyrins Phthalocyanines 2000, 4, 635.

23. Handa, M.; Suzuki, A.; Shoji, S.;Kasuga, K.; Sogabe, K. Inorg. Chim. Acta 1995, 230, 41.

24. Suzuki, T. ; Murayama, T.; Ono, H.; Otsuka, S.; Nozomi, M. (Mitsubishi Chemical Industries Co., Ltd., Japan). Eur. Pat. Appl. 1986, 29 pp. EP 180931, A2 19860514.

25. Goedken, V.L.; Dessy, G.; Ercolani, C.; Fares, V.; Gastaldi, I. Inorg. Chem. 1985, 24, 991.

26. Kleinwachter, J.; Subramanian, L.R.; Hanack, M. J. Porphyrins Phthalocyanines 2000, 4, 498.

27. Gires, F. I. IEEE J. Quant. Electr. 1966, QE-2, 624.

28. Shirk, J.J.; Pong, R.G.S.; Flom, S.R.; Heckmann, H.; Hanack, M. J. Phys. Chem. A 2000, 104, 1438 .

29. Hercher, M. Appl. Opt. 1967, 6, 947.

30. Perry, J.W.; Mansour, K.; Lee, I.Y.S.; Wu, X.L.; Bedworth, P.V.; Chen, C.T.; Ng, D.; Marder, S.R.; Miles, P.; Wada, T.; Tian, M.; Sasabe, H. Science 1996, 273, 1533.

31. Buckley, A.; Choe, E.; De Martino, R.; Leslie, T.; Nelson, G.; Stamatoff, J.; Stuetz, D.; Yoon, H. Polym. Mat. Sci. Eng. 1986, 54, 502.

32. Lever, A.B.P.; Pickens, S.R.; Minor, P.C.; Licoccia, S.; Ramaswamy, B.S.; Magnell, K. J. Am. Chem. Soc. 1981, 103, 6800.

33. Wei, T.H.; Hagan, D.J.; Sence, M.J.; Van Stryland, E.W.; Perry, J.W.; Coulter, D.R. Appl. Phys. B: Photophys. Laser Chem. 1992, B54, 46.

34. Bredas, J.L.; Adant, C.; Tackx, P.; Persoons, A. Chem. Rev. 1994, 94, 243.

35. Jaffe, H.H.; Orchin, M. Theory and Application of Ultraviolet Spectroscopy, Wiley: New York, 1962.

(C) 2003 by MDPI (http://www.mdpi.org). Reproduction for noncommercial purposes permitted. 